\title{
Effect of contact angle hysteresis on thermocapillary droplet actuation
}

\author{
Jian Z. Chen \\ Department of Electrical Engineering, Princeton University, Princeton, New Jersey 08544 \\ Sandra M. Troian ${ }^{\text {a) }}$ and Anton A. Darhuber \\ Microfluidic Research and Engineering Laboratory, Department of Chemical Engineering, \\ Princeton University, Princeton, New Jersey 08544 \\ Sigurd Wagner \\ Department of Electrical Engineering, Princeton University, Princeton, New Jersey 08544
}

(Received 31 March 2004; accepted 24 September 2004; published online 15 December 2004)

\begin{abstract}
Open microfluidic devices based on actuation techniques such as electrowetting, dielectrophoresis, or thermocapillary stresses require controlled motion of small liquid droplets on the surface of glass or silicon substrates. In this article we explore the physical mechanisms affecting thermocapillary migration of droplets generated by surface temperature gradients on the supporting substrate. Using a combination of experiment and modeling, we investigate the behavior of the threshold force required for droplet mobilization and the speed after depinning as a function of the droplet size, the applied thermal gradient and the liquid material parameters. The experimental results are well described by a hydrodynamic model based on earlier work by Ford and Nadim. The model describes the steady motion of a two-dimensional droplet driven by thermocapillary stresses including contact angle hysteresis. The results of this study highlight the critical role of chemical or mechanical hysteresis and the need to reduce this retentive force for minimizing power requirements in microfluidic devices. (C) 2005 American Institute of Physics. [DOI: 10.1063/1.1819979]
\end{abstract}

\section{INTRODUCTION}

Actuation of very small droplets for microfluidic applications can be accomplished by a number of different methods. The flow of liquid within enclosed channels of glass, silicon, or plastic is possible by use of electrophoretic or electro-osmotic forces, ${ }^{1}$ electrohydrodynamic, ${ }^{2}$ pneumatic, ${ }^{3}$ or thermocapillary pumping, ${ }^{4}$ centrifugation, ${ }^{5}$ and magnetohydrodynamics. ${ }^{6}$ Some of these techniques rely critically on the electrical or magnetic properties of the liquid. By contrast, many fewer actuation techniques are currently available for free-surface flows, whereby liquid streams or droplets are propelled across the exterior surface of a solid substrate. Recent experiments with open microfluidic devices have demonstrated that electrowetting, ${ }^{7-12}$ dielectrophoresis, ${ }^{13}$ and thermocapillary modulation of surface stresses ${ }^{14-16}$ can successfully induce shape changes as well as liquid migration. Electrowetting is also being used to actuate optofluidic elements like tunable optical fibers ${ }^{17}$ and tunable liquid lenses. ${ }^{11}$ Our group has been focusing during the past few years on thermocapillary actuation by coupling chemical patterning of substrates (for definition of fluidic pathways and networks) with embedded microheater arrays, which allow selective tuning of local shear stresses at the air interface for generating motion. ${ }^{14-16}$ The small thermal conductivity of the glass substrate ensures large but localized thermal gradients ${ }^{18}$ for controlling various fluidic functions with good positional resolution. The number of manipulations possible by application of different surface thermal maps includes control of droplet speed and direction, trapping, splitting, coalescence

\footnotetext{
a) Author to whom correspondence should be addressed; electronic mail: stroian@princeton.edu
}

and mixing of different liquid samples, and liquid metering.

Previous modeling efforts for cases involving a constant thermocapillary stress have successfully reproduced the results of experiments on liquid propulsion ${ }^{14}$ and mixing ${ }^{19}$ in continuous microstreams. Actively tunable manipulation of discrete liquid elements (i.e., droplets), however, requires spatially nonuniform thermal maps, a situation more difficult to model theoretically. The contact line, which defines the intersection boundary of the gas, liquid, and solid phases, gives rise to contact angle hysteresis, which can pin a liquid sample in place even in the presence of an external driving force. This retentive force, when caused by surface imperfections like chemical heterogeneities, ${ }^{20,21}$ asperities, and roughness $^{22,23}$ is called mechanical hysteresis. Chen et al. ${ }^{24}$ have shown, however, that even surfaces that are smooth and chemically homogeneous can exhibit large contact angle hysteresis. In this case, the retentive force is caused by molecular reorganization and relaxation upon contact of the liquid and solid phases, especially when surfaces contain both polar and nonpolar groups. This type of hysteresis, which often occurs on substrates treated with a monolayer coating, is known as chemical hysteresis. In either case, contact angle hysteresis, whose total influence is proportional to the length of the contact line, can generate a significant opposing force as the ratio of contact line arc length to liquid surface area increases with decreasing droplet size. This is an issue of considerable concern in the development of microfluidic devices.

There exist only phenomenological treatments of this retentive force for inclusion in hydrodynamic models of droplet motion. The focus of this work is to provide a simple framework for quantifying the threshold force necessary for 
droplet mobilization and the propulsion speed after depinning as a function of droplet size, thermal gradient, and liquid parameters. These studies hopefully provide additional insight into which mechanisms most strongly influence droplet pinning and flow speed for thermocapillary actuation. In some previous experimental studies, we used discrete microheater arrays fabricated on glass samples ${ }^{15,16}$ in order to maximize droplet speed. In this study, we used single-crystal silicon substrates for generating a smooth and constant thermocapillary stress. The higher thermal conductivity of silicon was used to create a linear thermal profile (i.e., constant shear stress) which greatly simplifies comparison to theoretical predictions.

\section{PRIOR STUDIES OF THERMOCAPILLARY PROPULSION}

The propulsion of small droplets by a constant thermocapillary force has previously been investigated by a number of groups. ${ }^{15,16,25-30}$ Brochard $^{26}$ explored the analogy between droplet migration due to a small variation in the substrate surface energy by chemical means and a small variation in the liquid surface tension by thermal means. Droplet deformation during transport was assumed to be negligible. In the first case, the substrate surface energy was varied by modifying the spreading coefficient, $S=\gamma_{s v}-\gamma_{s l}$ $-\gamma_{l v}$, where $\gamma_{i j}$ refers to the interfacial tension between the solid $(s)$, liquid $(l)$, and vapor $(v)$ phases. Under isothermal conditions, the droplet moves in the direction of increasing values of $S=\gamma_{s v}-\gamma_{s l}$, i.e., toward the more wetting surface. A similar analysis for thermocapillary migration, neglecting any thermal variation in $\gamma_{s v}-\gamma_{s l}$, showed that the droplet moves towards the cooler surface. Brzoska et al. ${ }^{27}$ conducted a number of experiments on thermocapillary flow using droplets of various poly(dimethylsiloxane) oils on a hydrophobic surface. The droplets, which ranged in length from about $2-10 \mathrm{~mm}$, were measured to have contact angles between $11^{\circ}-13^{\circ}$. The thermal gradients applied to the silicon substrates were rather small and less than $1{ }^{\circ} \mathrm{C} / \mathrm{mm}$. The droplet speed was shown to depend on the applied thermal gradient, the initial radius of the deposited droplet and the oil viscosity (assumed constant). Contact angle hysteresis caused pinning below a critical droplet radius for a fixed thermal gradient. Above this threshold, the droplet speed was linearly proportional to the applied thermal gradient and inversely proportional to the liquid viscosity. Ford and Nadim $^{28}$ presented a hydrodynamic description of droplet pinning and migration for a two-dimensional (2D) geometry based on a force balance at the liquid-solid interface within the lubrication approximation. This approximation is valid provided the characteristic height to length ratio is very small. Their analysis for a constant thermocapillary stress allows for small shape distortion by capillary and thermocapillary forces and a Navier slip condition to resolve the wellknown stress singularity at a moving contact line. ${ }^{31}$ Under these assumptions, the droplet speed was shown to depend strongly on the slip coefficient, a parameter which is exceedingly difficult to measure experimentally. Smith ${ }^{29}$ provided a complete hydrodynamic analysis within the lubrication ap- proximation for a $2 \mathrm{D}$ droplet subject to a constant thermocapillary stress. In addition to the Navier slip condition, he implemented a dynamic boundary condition for which the contact angle depends on the speed of the contact line. He too neglected any variation of the liquid viscosity with temperature. The balance of thermocapillary and viscous stresses gives rise to two time-independent solutions: a stationary droplet with internal circulation or a droplet translating at constant speed with no change in shape. Yarin et al. ${ }^{30}$ examined the thermocapillary migration of axisymmetric alkane droplets clinging to a partially wetting cylindrical fiber. The experimental measurements of droplet position with time were compared to a simplified model that neglected droplet deformation due to capillary pressure gradients and contact angle hysteresis but did incorporate the change in viscosity with temperature. Most recently, Tseng et al. ${ }^{25}$ used a linear array of distributed heating elements embedded within a glass substrate to induce thermocapillary motion in small droplets, as in previous studies. ${ }^{15,16}$ Details of the physical mechanisms included in their hydrodynamic analysis or the boundary conditions used in obtaining the final solutions, however, were not provided.

There are two major difficulties in attempting to quantify thermocapillary propulsion of discrete droplets on surfaces which are not completely wetting, i.e., surfaces for which there is no precursor film ahead of the moving contact line. ${ }^{31}$ First, the physical mechanisms controlling the fluid dynamics at the moving contact line are not well understood and the appropriate boundary conditions not well established. Most analyses therefore rely on phenomenological descriptions of contact line motion and viscous dissipation near the contact line. Secondly, if mechanical hysteresis is to be minimized, then the use of smooth, high-quality surfaces is essential for obtaining good reproducibility and droplet migration with small applied driving force and minimal power input.

In this work, we examine thermocapillary migration of droplets consisting of various organic liquids on silicon surfaces chemically treated with a silanizing monolayer. Alkyltrichlorosilanes self-assembling monolayers were grafted onto oxidized silicon wafers to produce highly reproducible surfaces containing a small number of observable surface defects. The chemical treatment generates partially wetting surfaces with liquid contact angles in the range $13^{\circ}<\theta$ $<41^{\circ}$. Droplet mobilization requires application of thermal gradients in the range $2{ }^{\circ} \mathrm{C} / \mathrm{mm}<d T / d x$ $<4.5^{\circ} \mathrm{C} / \mathrm{mm}$. We present a simple generalized model for evaluating the magnitude of the threshold depinning force caused by contact angle hysteresis based on earlier work by Ford and Nadim. ${ }^{28}$ This model, which quantitatively supports the experimental results, clearly shows that contact angle hysteresis plays a significant role in establishing the conditions for droplet depinning. Droplets can only be mobilized above a threshold radius for a fixed thermal gradient ${ }^{27}$ or above a threshold thermocapillary stress for a fixed droplet radius. The effects of these two limiting variables are combined by evaluating the threshold depinning force per unit length required for droplet mobilization. These results provide some practical guidelines for the design of microfluidic chips based on thermocapillary actuation. 

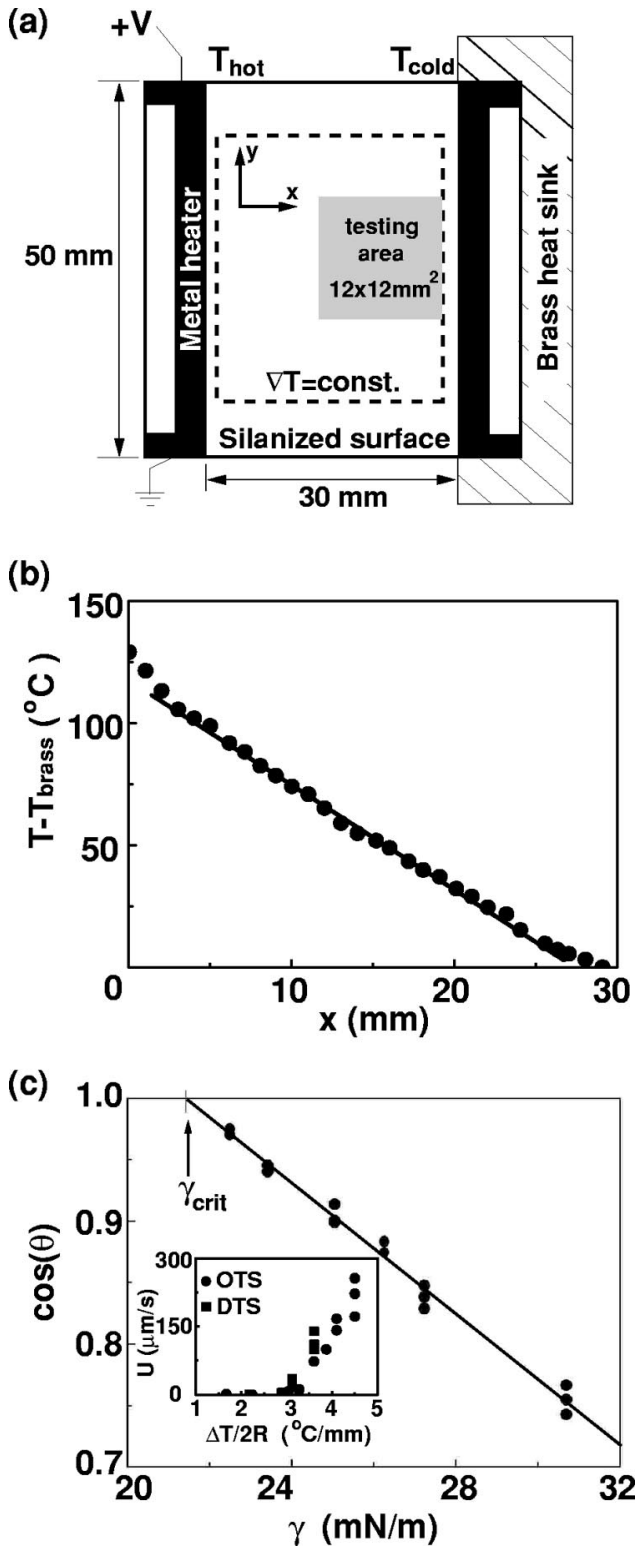

FIG. 1. (a) Experimental layout for generating a constant thermal gradient $d T / d x$ on a silanized silicon wafer. (b) Results of surface thermocouple measurements for an applied gradient of $-4.21^{\circ} \mathrm{C} / \mathrm{mm}$. (c) Zisman plot for droplets on a DTS-coated silicon substrate. The liquids used to obtain the critical surface tension, $\gamma_{\text {crit }}$, were $n$-decane, n-dodecane, n-tetradecane, n-hexadecane, and squalane. Inset: Measurements of the droplet speed (volume $=5 \mu \mathrm{l}$ ) as a function of the applied thermal gradient, $\Delta T / 2 R$, on DTS- and OTS-coated substrates.

\section{EXPERIMENTAL SETUP AND SAMPLE PREPARATION}

As shown in Figs. 1(a) and 1(b), a constant thermal gradient was generated along the solid surface by powering a metal heater stripe at one end of the sample and circulating coolant through a brass heat sink at the opposite end. The intrinsic high thermal conductivity of single-crystal silicon (Si) wafers generates a linear surface temperature distribution since the heat flux through the silicon dominates any thermal losses due to natural convection under ambient conditions. Figure 1(b) shows a typical temperature profile as measured with an HH21 Omega thermocouple. The diameter of the spherical probe ( $K$-type $\mathrm{Ni}-\mathrm{Cr}$ ) was $0.127 \mathrm{~mm}$ and its resolution $\pm 0.1{ }^{\circ} \mathrm{C}$. Measurements were taken at 1 -mm intervals along the entire silicon surface. The temperature gradient was found to be constant except within about $3 \mathrm{~mm}$ of the heating stripe and heat sink. The experiments on droplet mobilization were therefore confined to a central $12 \mathrm{~mm}$ $\times 12 \mathrm{~mm}$ region within which $d T / d x$ was constant [grey region shown in Fig. 1(a)]. Top views of stationary or moving droplets (travel distance $\Delta x=4 \mathrm{~mm}$ ) were recorded with a Pulnix TM1040 CCD camera connected to an Olympus BX-60 microscope. For each image acquired, the position of the advancing and receding contact lines (corresponding to the front and back end of a moving droplet) was extracted and used to determine the droplet speed as a function of droplet radius and applied thermal gradient.

All liquids used, namely, squalane $\left(\mathrm{C}_{30} \mathrm{H}_{62}, 99 \%\right)$, $n$-decane $\left(\mathrm{C}_{10} \mathrm{H}_{22}, 99 \%\right), n$-dodecane $\left(\mathrm{C}_{12} \mathrm{H}_{26}, 99 \%\right)$, $n$-tetradecane $\left(\mathrm{C}_{14} \mathrm{H}_{30}, 99 \%\right)$, and $n$-hexadecane $\left(\mathrm{C}_{16} \mathrm{H}_{34}\right.$, 99\%), were purchased from Sigma-Aldrich and filtered through a $0.2-\mu \mathrm{m}$ glass syringe filter to eliminate macroscopic particulates. Table I lists the corresponding parameters relevant to this study. A Hamilton 1700 series digital syringe with a teflon-coated needle (10- $\mu \mathrm{l}$ maximum capacity and $0.05-\mu$ l resolution) was used to deposit droplets on the silanized substrates.

The quality of the grafted monolayers depends strongly on the process environment, including the temperature, ${ }^{34,35}$ total immersion time ${ }^{36-38}$ solvent type, ${ }^{38,39}$ silane concentration, ${ }^{38}$ water content, ${ }^{36,37}$ and roughness of the native oxide layer. ${ }^{37}$ Given the number of variables controlling the coating quality, it is no surprise that the grafting process is extremely sensitive to environmental conditions. We spent a considerable amount of time identifying process-

TABLE I. Physical properties of liquids (at $25{ }^{\circ} \mathrm{C}$ ) used in this study, where $\rho$ is the density $\left(\mathrm{kg} / \mathrm{m}^{3}\right), \gamma_{l v}$ is the surface tension $(\mathrm{mN} / \mathrm{m}), d \gamma_{l v} / d T$ is the thermal coefficient of surface tension $\left(\mathrm{mN} / \mathrm{m}{ }^{\circ} \mathrm{C}\right)$, and $\mu$ is the viscosity (mPa s). These material constants were obtained from Refs. 32 and 33. The last two rows represent values for the threshold retentive force, $(\tau R)_{t}$, in $\mathrm{mN} / \mathrm{m}$ and the nondimensional force, $(\tau R)_{t} / \gamma_{l v}$, extracted from similar experimental measurements as in Fig. 6.

\begin{tabular}{cccccc}
\hline \hline & $n-\mathrm{C}_{10} \mathrm{H}_{22}$ & $n-\mathrm{C}_{12} \mathrm{H}_{26}$ & $n-\mathrm{C}_{14} \mathrm{H}_{30}$ & $n-\mathrm{C}_{16} \mathrm{H}_{34}$ & $\mathrm{C}_{30} \mathrm{H}_{62}$ \\
\hline$\rho$ & 726.1 & 745.2 & 759.2 & 770.0 & 809.0 \\
$\gamma_{l v}$ & 23.43 & 25.04 & 26.26 & 27.22 & 30.7 \\
$d \gamma_{l v} / d T$ & -0.092 & -0.088 & -0.086 & -0.084 & -0.050 \\
$\mu$ & 0.838 & 1.383 & 2.128 & 3.032 & 29.51 \\
$(\tau R)_{t}$ & 0.31 & 0.42 & 0.58 & 0.60 & 0.32 \\
$(\tau R)_{t} / \gamma_{l v}$ & 0.0134 & 0.0179 & 0.0228 & 0.0236 & 0.0121 \\
\hline \hline
\end{tabular}


ing parameters which could be suitably accommodated to our laboratory environment based on the key factors mentioned above.

All substrates were prepared from $n$-type $\operatorname{Si}(100)$ wafers. The native surface oxide was first removed with a 1:10 buffered oxide etch solution. The substrate was then rinsed with distilled doubly de-ionized water (18 $\mathrm{M} \Omega$, Hydro Services) and immediately cleaned with a mixture of $\mathrm{H}_{2} \mathrm{O}_{2}$ and $\mathrm{H}_{2} \mathrm{SO}_{4}$ (1:2 volume ratio) for $5 \mathrm{~min}$. During this step, a thin wetoxide layer was formed whose surface roughness is far less than thermally grown oxide layers. ${ }^{37}$ This wet-oxide film provides the necessary layer for grafting the trichlorosilane head groups. Layers of 300-nm Ti and 5-nm Au were deposited with a Denton electron-beam evaporator at $10^{-6}$ torr base pressure. This deposition step was followed by photolithography and lift-off to define the geometry of the metal heating stripe. After the lift-off process, the substrates were rinsed with acetone and isopropanol and then blown dry in a filtered nitrogen stream. To remove any residual organic surface contamination, the substrates were further cleaned by applying a $200-\mathrm{mW}$ oxygen plasma for $2 \mathrm{~min}$ before immersion in the alkyltrichlorosilane solution. The time required for removal of the substrate from the plasma chamber to immersion in the silanizing solution was less than $1 \mathrm{~min}$.

This process was used to form hydrophobic coatings of dodecyltrichlorosilane (DTS, 99\%, Aldrich) or octadecyltrichlorosilane (OTS, 95\%, Aldrich). The reactive solution consisted of 1-mM DTS or OTS and dodecane $(99+\%$, anhydrous, Aldrich). The deposition proceeded under a dry $\mathrm{N}_{2}$ environment at $9{ }^{\circ} \mathrm{C}$ for $1.5 \mathrm{~h}$ for DTS coatings and at room temperature for $24 \mathrm{~h}$ for OTS coatings. These deposition temperatures were selected based on the transition temperatures reported for optimal alkyltrichlorosilane coatings. ${ }^{34,35}$ The deposition procedure was terminated by withdrawing the Si wafer from the reactive solution, rinsing it with trichloroethylene (TCE, anhydrous, Aldrich), and sonicating the substrate in a TCE solution for $10 \mathrm{~min}$. The samples were finally rinsed with isopropanol, blown dry in a filtered nitrogen stream, and stored in a vacuumed desiccator for later use.

This process was found to yield reproducible coatings of high quality with few observable surface defects. The critical surface tension of the coated substrates was measured by the Zisman method, which is widely used for characterizing surface energies. ${ }^{40} \mathrm{~A}$ Zisman plot consists of measurements of $\cos (\theta)$ versus $\gamma_{l_{v}}$ on a given substrate for various liquids, where $\theta$ denotes the static contact angle on a level surface. Extrapolation of the data to the intercept $\cos (\theta)=1$ yields the critical surface tension corresponding to the substrate surface. Within experimental error, the critical surface tension of the DTS- and OTS-coated substrates was measured to be $21.3 \pm 0.3 \mathrm{mN} / \mathrm{m}$. This value was obtained by a least-squares fit of the data shown in Fig. 1(c) using the values, $\gamma_{l v}$, listed in Table I. This measured value is slightly higher than the value obtained by Brzoska et al. $(20.5 \pm 0.5 \mathrm{mN} / \mathrm{m})$ for their surface coatings. ${ }^{35}$

The difference between the advancing and receding contact angle, $\theta_{a}-\theta_{r}$, is normally used to quantify the retentive force for droplet mobilization. This difference, measured by the tilting plane method, ${ }^{41}$ was found to be about $9^{\circ} \pm 2^{\circ}$ for

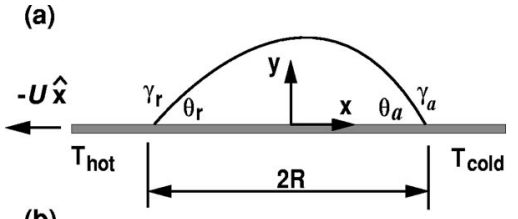

(b)

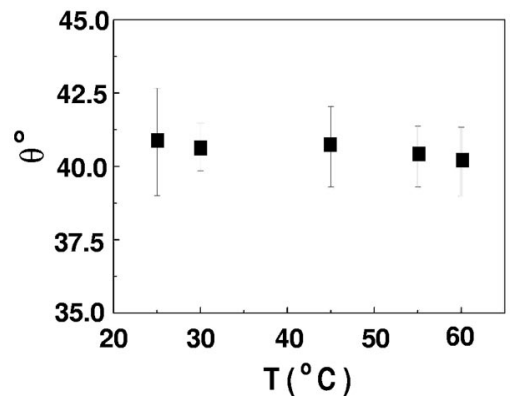

FIG. 2. (a) Geometry corresponding to the steady motion of a 2D droplet on a hydrophobic surface in a moving frame of reference. The speed $U$ represents the height-averaged speed for thermocapillary propulsion in the laboratory frame. The subscripts $a$ and $r$ denote the advancing and receding edges of the droplet. (b) Measurements of the static contact angle for a squalane droplet resting on a uniformly heated DTS-coated substrate.

water, $1^{\circ}-3^{\circ}$ for squalane, and less than $2^{\circ}$ for the alkane liquids. We were therefore able to achieve reproducible droplet motion with application of a modest thermal gradient. The inset in Fig. 1(c) shows representative droplet speeds achieved on DTS- and OTS-coated substrates as a function of increasing thermal gradient, $d T / d x=\Delta T / 2 R$, where $\Delta T$ is the temperature difference across a droplet of length $2 R$.

\section{THEORETICAL DESCRIPTION}

To determine the validity of the approximations used below, we estimated typical values of the Peclet (Pe $\left.=U h_{c} \rho c_{p} / k_{\text {liq }}\right)$ and Biot $\left(\mathrm{Bi}=h_{\text {conv }} h_{c} / k_{\text {liq }}\right)$ numbers based on the maximum droplet height, $h_{c}$, and speed, $U$, obtained with our apparatus. From the following estimates $-0.5 \leqslant h_{c}$ $\leqslant 1.1 \mathrm{~mm}, \quad 0 \leqslant U \leqslant 300 \mu \mathrm{m} / \mathrm{s}$, liquid heat capacity $c_{p}$ $\approx 1.5 \mathrm{~kJ} / \mathrm{kg}{ }^{\circ} \mathrm{C},{ }^{32}$ liquid thermal conductivity $k_{l i q}$ $\approx 0.14 \mathrm{~W} / \mathrm{m}{ }^{\circ} \mathrm{C},{ }^{32}$ and heat transfer coefficient based on natural convection $h_{\text {conv }} \approx 7.5 \mathrm{~W} / \mathrm{m}^{2}{ }^{\circ} \mathrm{C}$-these characteristic numbers were determined to be $\mathrm{Pe} \lesssim 2.8$ and $0.02 \lesssim \mathrm{Bi}$ $\lesssim 0.06$. The low values of $\mathrm{Bi}$ confirm that vertical variations in temperature were negligible for small and slowly moving droplets. For larger or faster droplets, however, $\mathrm{Pe} \sim \mathcal{O}(1)$, which suggests a nonconstant stress along the air-liquid interface. The model described below incorporates this possibility by allowing a nonuniform surface stress $\tau(x)$. Estimates of the lubrication parameters, $\left(h_{c} / R\right)^{2} \approx 0.09$, and $h_{c} / R \times \operatorname{Re} \approx 0.04$, where $\operatorname{Re}=\rho U h_{c} / \mu$ is the Reynolds number and $R$ the equilibrium radius of a droplet resting on a substrate, justify the use of the lubrication approximation. ${ }^{42}$

Consider a 2D droplet moving at constant velocity, $U \hat{e}_{x}$, in response to a positive thermocapillary stress, $\tau(x)$ $=d \gamma_{l v} / d T \times d T / d x$, where $T(x)$ is the liquid surface temperature. In the frame of reference moving with the average speed of the liquid, the droplet boundaries remain stationary and the substrate moves to the left with velocity $-U \hat{e}_{x}$, as shown in Fig. 2(a). For convenience, we hereon designate 
the liquid-vapor surface tension, $\gamma_{l v}$, by $\gamma(x)$. The contact angle and surface tension corresponding to the advancing $(a)$ and receding $(r)$ edges of the droplet are designated by $\theta_{a}, \theta_{r}$, $\gamma_{a}$, and $\gamma_{r}$, respectively.

In Fig. 2(b) is plotted the measurements of the static contact angle versus increasing temperature for a droplet of squalane resting on a uniformly heated DTS substrate. Within experimental error, there was no systematic deviation in contact angle despite the large range in temperature $\left(25-60{ }^{\circ} \mathrm{C}\right)$. This measurement is consistent with the small values, $d \theta / d T$, reported in the literature $(0.01-0.1$ $\left.\mathrm{deg} /{ }^{\circ} \mathrm{C}\right) .{ }^{43}$ Any changes in the value $\theta_{a}-\theta_{r}$ were therefore attributed to contact angle hysteresis although this assumption does not figure explicitly into the following derivation. For moving contact lines, the advancing contact angle is known to increase with contact line speed. ${ }^{44-46}$ The derivation outlined below implicitly accommodates this dynamic variation in contact angle through corresponding changes in the droplet shape $h(x)$.

Within the lubrication approximation for a Newtonian liquid, the Navier-Stokes equations in the moving reference frame reduce to

$$
\begin{aligned}
& \frac{\partial P}{\partial x}=\mu \frac{\partial^{2} u}{\partial y^{2}}, \\
& \frac{\partial P}{\partial y}=-\rho g .
\end{aligned}
$$

The two boundary conditions on the $x$ component of the velocity field, $u(x, y)$, are given by

$$
\begin{aligned}
& \mu\left(\frac{\partial u}{\partial y}\right)_{y=h(x)}=\tau(x)=\frac{d \gamma}{d T}\left(\frac{d T}{d x}\right)_{y=h(x)}, \\
& u(y=0)=-U+b\left(\frac{\partial u}{\partial y}\right)_{y=0} .
\end{aligned}
$$

Here, $P$ denotes the pressure field, $\mu$ the liquid viscosity, $\rho$ the liquid density, $g$ the gravitational acceleration constant, $h(x)$ the droplet shape, and $b$ the Navier slip length. ${ }^{28}$ The plane $y=0$ designates the liquid-solid interface; the vapor phase is assumed passive and all evaporative effects are ignored.

The overall pressure affecting fluid motion consists of the capillary pressure caused by curvature of the liquid-vapor interface and possible hydrostatic pressure. Accordingly,

$$
P(x, y)=\rho g[h(x)-y]-\gamma(x) \frac{d^{2} h}{d x^{2}}\left[1+\left(\frac{d h}{d x}\right)^{2}\right]^{-3 / 2} .
$$

The solution to Eq. (1) is therefore given by

$$
u(x, y)=\frac{1}{2 \mu} \frac{\partial P}{\partial x}\left(y^{2}-2 h y-2 b h\right)+\frac{\tau}{\mu}(y+b)-U,
$$

where the droplet travel distance is assumed small and the viscosity therefore constant. A stationary droplet requires that the volumetric flux (per unit length in $\hat{z}$ ), $Q$, identically vanish, i.e.,

$$
\int_{0}^{h(x)} u(x, y) d y=0
$$

The pressure gradient satisfying this constraint is given by

$$
\frac{\partial P}{\partial x}=\frac{\tau(h / 2+b)-\mu U}{h^{2} / 3+b h} .
$$

Furthermore, provided that thermal variations in $\gamma_{s v}$ and $\gamma_{s l}$ are negligible in comparison to $d \gamma / d T$, the equilibrium force balance $^{28}$ at $y=0$ requires that

$$
\int_{-R}^{R} \mu\left(\frac{\partial u}{\partial y}\right)_{y=0} d x+\gamma_{r} \cos \theta_{r}-\gamma_{a} \cos \theta_{a}=0 .
$$

Substitution of the velocity field, $u(x, y)$, into Eq. (9) yields an expression for the droplet speed $U$, namely,

$$
U=\frac{1}{6 \mu R J}\left[\int_{-R}^{R} \frac{\tau h / 2}{h+3 b} d x-\left(\gamma_{r} \cos \theta_{r}-\gamma_{a} \cos \theta_{a}\right)\right],
$$

where $J$ is defined as

$$
J=\frac{1}{2 R} \int_{-R}^{R} \frac{d x}{h(x)+3 b} .
$$

Equation (10) can be simplified by assuming that the slip length, $b$, is orders of magnitude smaller than the local droplet thickness $h(x)$, a valid assumption except at the edges where the droplet thicknesses approaches molecular dimensions. ${ }^{47}$ Furthermore, since most liquids maintain a constant value of $d \gamma / d T$ over the range of interest, Eq. (10) reduces to

$$
U=\frac{1}{6 \mu J}\left(\frac{d \gamma}{d T} \frac{\Delta T}{2 R}+\frac{\gamma_{a} \cos \theta_{a}-\gamma_{r} \cos \theta_{r}}{R}\right),
$$

where $\Delta T=|T(x=+R)-T(x=-R)|$. The second term in Eq. (12) can be further expanded by noting that $\gamma_{a}=\gamma_{r}$ $+(\Delta T|d \gamma / d T|)$ such that

$$
U=\frac{1}{6 \mu J}\left[\left(1+2 \cos \theta_{a}\right)\left|\frac{d \gamma}{d T}\right| \frac{\Delta T}{2 R}-\frac{\gamma_{r}\left(\cos \theta_{r}-\cos \theta_{a}\right)}{R}\right] .
$$

As evident, droplet propulsion is established by the competition between the thermocapillary stress and contact angle hysteresis. Our experimental data were compared to the predictions of Eq. (13) where $\theta_{a}$ and $\theta_{r}$ were obtained from tilted plate measurements. This representation for the forces controlling droplet motion differs from the analysis of Brzoska et al., where the hysteresis term was incorporated as an ad hoc force and the surface tension at the advancing and receding edges was assumed equal. ${ }^{27}$ These two simplifications lead to an expression for the droplet speed different from Eq. (13).

The limit $U=0$ reveals the threshold force required for droplet depinning, namely, 


$$
\begin{aligned}
(\tau R)_{t} & \equiv\left(\left|\frac{d \gamma}{d T}\right| \frac{\Delta T}{2 R}\right) R \\
& =\frac{\gamma_{r}\left(\cos \theta_{r}-\cos \theta_{a}\right)}{1+2 \cos \theta_{a}}=\frac{\gamma_{a}\left(\cos \theta_{r}-\cos \theta_{a}\right)}{1+2 \cos \theta_{r}} .
\end{aligned}
$$

This threshold value only depends on the local value of the contact angle and surface tension at the advancing and receding edges but nowhere inbetween. In addition, motion requires a critical droplet radius, $R_{\min }$, for a given value of $\Delta T$ or a critical value of $\Delta T$ for a given droplet radius. The derivation leading to Eq. (14) allows for a variable shear stress $\tau(x)$. For a constant shear stress, Eq. (10) can be integrated directly to give

$$
U=\frac{\tau}{6 \mu J}-\frac{\tau b}{2 \mu}+\frac{\gamma_{a} \cos \theta_{a}-\gamma_{r} \cos \theta_{r}}{6 \mu R J},
$$

as first derived by Ford and Nadim. ${ }^{28}$ Equation (15) accounts for droplet deformation due to capillary and hydrostatic forces, which are intrinsically incorporated through the spatial variation in droplet shape $h(x)$.

\section{EXPERIMENTAL RESULTS AND DISCUSSION}

Figure 3(a) shows a typical measurement of droplet position versus time for squalane droplets on a DTS-coated surface. In this example, there were no significant surface defects along the travel distance. The droplets were deposited after the silicon wafer had thermally equilibrated to produce a constant thermal gradient. The droplet achieved a constant speed almost immediately after deposition and maintained this value over a distance of $4 \mathrm{~mm}$. The change in liquid viscosity during the motion was ignored since the travel distance was rather small. The upper-left inset in Fig. 3(a) depicts a side view image of the droplet shape. The circle which is superimposed on the droplet profile demonstrates that there was little distortion during motion, as expected for small thermal gradients and small contact angle hysteresis. The images on the right depict top views of the moving droplet at three different times. The elongation ratio $l_{x} / l_{y} \lesssim 1.04$ confirmed that the droplet footprint was essentially circular in shape.

Figure 3(b) shows a typical measurement of droplet position versus time for a squalane droplet on a DTS-coated substrate which contained one significant surface defect. As the droplet became pinned on the defect, it suffered some elongation; however, upon overcoming the obstacle, the receding end snapped back in place to produce a nearly circular footprint. The droplet was observed to undergo pinning not while advancing onto the defect but only when receding from it. This suggests that the defect was more wetting than the surrounding areas, an observation in agreement with previous studies which have reported that receding angles are highly affected by the more polar regions (wettable patches) of a heterogeneous surface..$^{20,21}$

The motion of squalane droplets, which are nonvolatile under the environmental conditions specified, were compared with the theoretical prediction in Eq. (13). The measured speed versus droplet radius is plotted in Fig. 4(a) (open symbols). Tests were conducted on the same DTS substrate

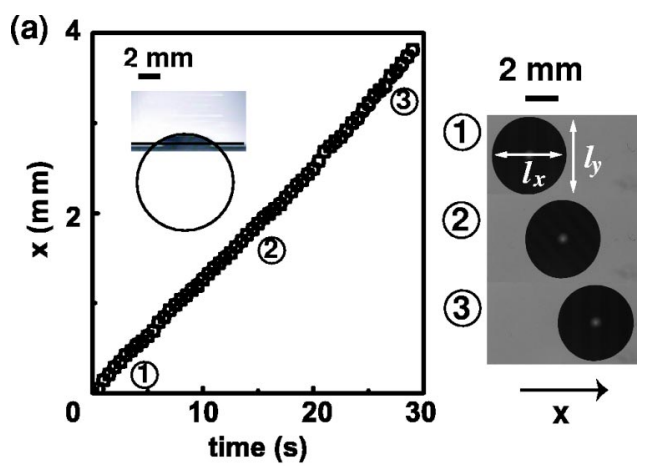

(b)

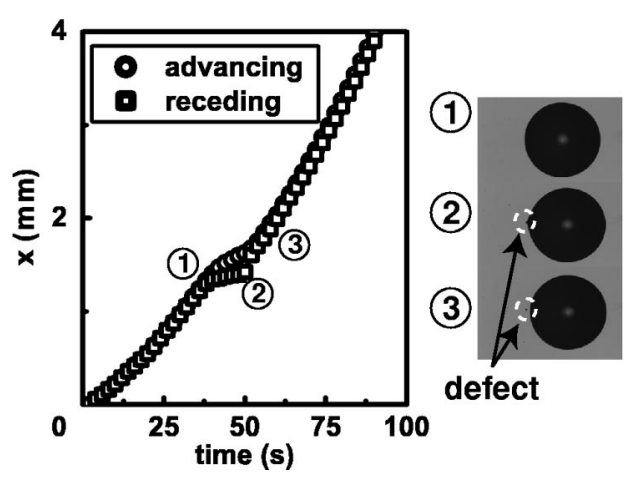

FIG. 3. (a) Experimental measurements of droplet position vs time for a squalane droplet (volume $=5 \mu \mathrm{l}$ ) subject to a thermal gradient $d T / d x=$ $-3.12{ }^{\circ} \mathrm{C} / \mathrm{mm}$. The coordinate values of the receding contact line have been shifted by $2 R$. The upper left inset shows the superposition of a circle onto a side view image of the moving droplet. The images to the right represent snapshots of the top view of moving droplets at three subsequent times. The ratio $l_{x} / l_{y}$ was used to measure elongation effects during propulsion. The level of distortion, however, was imperceptible indicating a surface with very low contact angle hysteresis. (b) Measured position of a squalane droplet $\left(\right.$ volume $=5 \mu \mathrm{l}$ ) subject to a thermal gradient of $d T / d x=-2.89{ }^{\circ} \mathrm{C} / \mathrm{mm}$. Due to the presence of a large surface defect, the advancing and receding contact lines moved at different speeds in the vicinity of the defect. The coordinate values of the receding contact line have been shifted by $2 R$.

using different thermal gradients and droplet volumes. The four solid lines represent best fits to the data based on Eq. (13) using a single set of fitting parameters. The liquid viscosity was determined from the average temperature within the testing area [see Fig. 1(a)] for each value of the applied thermal gradient. From the data for squalane given in Ref. 33 , the viscosities were estimated to be $28.3,36.2,39.8$, and $43.2 \mathrm{mPas}$ for thermal gradients $2.19,2.89,3.12$, and $3.60{ }^{\circ} \mathrm{C} / \mathrm{mm}$, respectively. The receding contact angles were fixed at the experimentally measured value of $41^{\circ}$. The advancing and receding values of the surface tension were estimated from the expression $\gamma_{a}=\gamma_{r}+2 R|d \gamma / d x|$. All droplet radii were obtained from video images and not estimated from the volume. The values for $J$ in Eq. (13) were estimated from side view images of the droplet profile, $h(x)$, as shown in the inset of Fig. 3. The two fitting parameters were therefore $b$ and $\theta_{a}$. The solid lines shown in Fig. 4(a) represent a best fit for $\theta_{a}-\theta_{r}=2.36^{\circ}$ and $b=3 \mathrm{~nm}$. This difference in contact angles falls well within the range measured by the tilting plane method. Overall, the numerical predictions describe the experimental data very well.

Figures 4(b) and 4(c) demonstrate the sensitivity of Eq. (13) to the two fitting parameters. For the physically mean- 


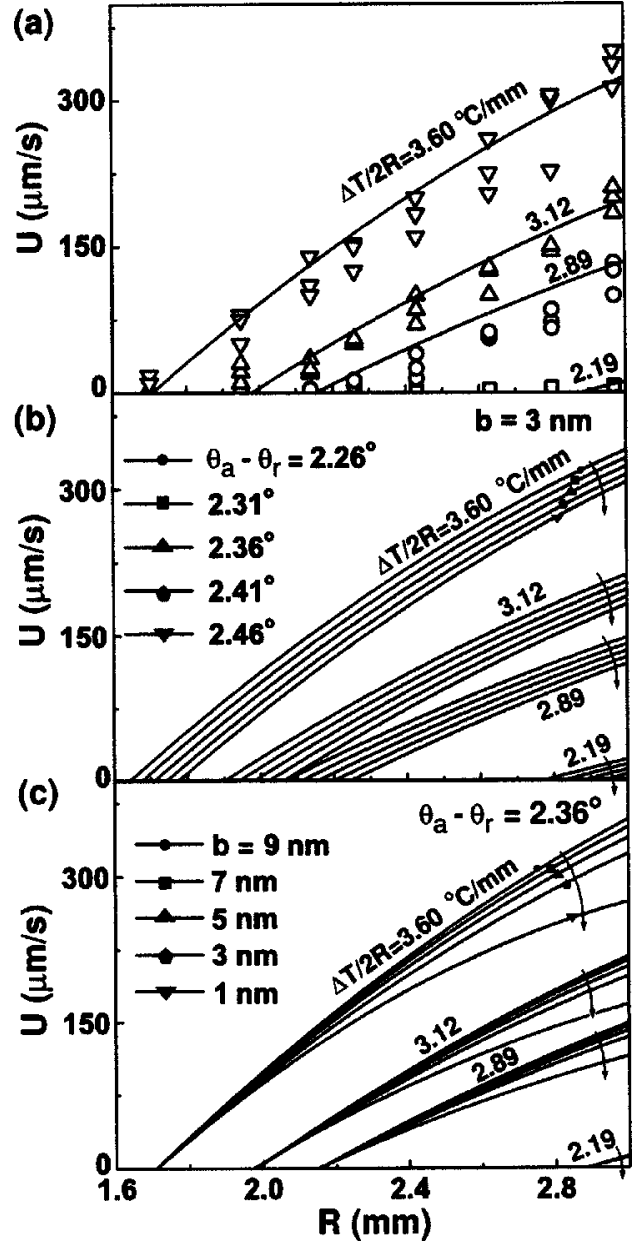

FIG. 4. (a) Measurements of droplet speed, $U$, vs droplet radius, $R$, for squalane (volume $=5 \mu \mathrm{l}$ ) on a DTS-coated surface for thermal gradient values $2.19 \leqslant \Delta T / 2 R \leqslant 3.60{ }^{\circ} \mathrm{C} / \mathrm{mm}$. Open symbols represent experimental data; solid lines are solutions to Eq. (13) with $\theta_{a}-\theta_{r}=2.36^{\circ}$ and $b=3 \mathrm{~nm}$. (b) Solutions to Eq. (13) for various values of $\theta_{a}-\theta_{r}$ at a fixed value of the slip coefficient $b=3 \mathrm{~nm}$. (c) Solutions to Eq. (13) for various values of $b$ at a fixed value of contact angle hysteresis $\theta_{a}-\theta_{r}=2.36^{\circ}$.

ingful range of hysteresis values considered here, the droplet speed was found to be much more sensitive to the value of the contact angle hysteresis than the value of the slip length. We therefore first fit the overall experimental trends by tuning $\theta_{a}-\theta_{r}$ and only then varying the value of the slip length. It is worth noting that smaller values of the slip length generate progressively larger influence since the slip length appears in the denominator of the integrand $J$. Physically, this reflects the fact that viscous dissipation near the contact line, where the film thickness approaches molecular dimensions, diverges logarithmically as $b \rightarrow 0 .^{31,48}$

As evident in Fig. 4(b), the droplet speed is highly sensitive to contact angle hysteresis: a small change in the value $\theta_{a}-\theta_{r}$ of only $0.2^{\circ}$ modifies the speed $U$ by over $16 \%$ for $U \approx 300 \mu \mathrm{m} / \mathrm{s}$ and by over $80 \%$ for $U \approx 50 \mu \mathrm{m} / \mathrm{s}$. Precise measurements of the dynamic contact angles and droplet distortion during transport present considerable experimental difficulties since the image resolution obtained by dynamic video capture is insufficient. Nevertheless, determination of the level of contact angle hysteresis by the tilted plane method produces good agreement between experimental measurements and Eq. (13).

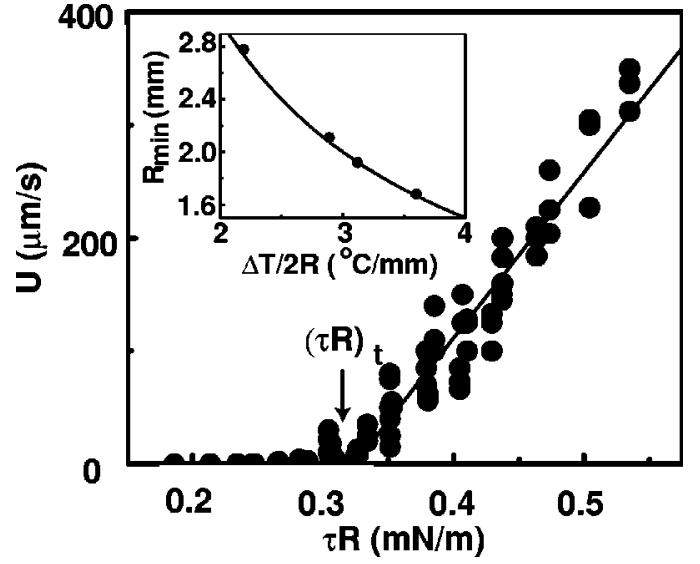

FIG. 5. Experimental data shown in Fig. 4 replotted as a function of the driving force $\tau R$. Onset of droplet migration provides an estimate of the threshold depinning force, $(\tau R)_{t}$. Inset: Measurements of the minimum droplet radius, $R_{\min }$, as a function of the applied thermal gradient $\Delta T / 2 R$. The fitted curve represents the solution $R_{\min }=(\tau R)_{t} /[|d \gamma / d T| \Delta T / 2 R]$.

Figure 5 represents the measured droplet speeds shown in Fig. 4(a) replotted as a function of $\tau R$. Despite the large scatter, the data collapse onto a common line over the entire parameter range investigated. The horizontal intercept represents the threshold depinning force, $(\tau R)_{t}=0.32 \mathrm{mN} / \mathrm{m}$ for squalane droplets on a DTS-coated substrate obtained from a (linear) least-squares fit. According to Eq. (13), since $J$ scales as $R^{-1}$, the droplet speed after depinning should scale linearly in $\tau R$, as observed. The results shown in the inset figure also confirm that the threshold radius, $R_{\min }$, is inversely proportional to the applied thermal gradient, $\Delta T / 2 R$, in agreement with Eq. (14). The predictions of Eq. (14) were also tested against the measured values of $R_{\min }$ reported by Brzoska et $a l^{27}$ using the parameter values provided in that reference. Assuming the values $\left|\cos \theta_{a}-\cos \theta_{r}\right|=1.5 \times 10^{-2}$, $\theta_{a}=13^{\circ}$, and $\gamma=21 \mathrm{mN} / \mathrm{m}$, the predictions of the threshold radii from Eq. (14) are given by 4.13, 2.58, 1.92, and $1.45 \mathrm{~mm}$ for corresponding thermal gradients $0.35,0.56$, 0.75 , and $1{ }^{\circ} \mathrm{C} / \mathrm{mm}$. The reported values were $3.71,2.29$, 1.88 , and $1.29 \mathrm{~mm}$ (measured from Brzoska's Fig. 2). ${ }^{27}$ Our predictions lie within $10 \%$ of these measured values.

In Fig. 6 is plotted the droplet speed as a function of the

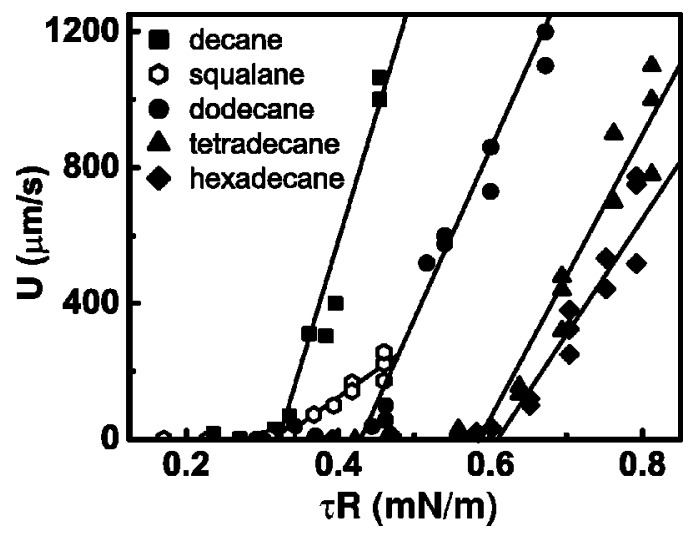

FIG. 6. Measurements of droplet speed as a function of the driving force, $\tau R$, on an OTS-coated substrate for the five liquids (volume $=5 \mu$ ) shown. The onset of droplet migration provides an estimate of the threshold depinning force $(\tau R)_{t}$. 
driving force, $\tau R$, for droplets of five different liquids moving on an OTS-coated substrate. The horizontal intercept represents the threshold retentive force per unit length based on a linear extrapolation (solid lines). In Table I can be found the extrapolated values, $\tau R / \gamma_{l v}$, which increase with linear alkane chain length. Squalane, which is a larger branched hydrocarbon molecule, deviates from this trend.

The motion of droplets of 1-propanol $\left(\gamma_{l v}\right.$ $=23.52 \mathrm{mN} / \mathrm{m}), \quad$ 1-butanol $\quad\left(\gamma_{l v}=25.17 \mathrm{mN} / \mathrm{m}\right) \quad$ and 1-hexanol $\left(\gamma_{l v}=26.05 \mathrm{mN} / \mathrm{m}\right)$ on OTS- and DTS-coated substrates was also examined. The surface tensions and contact angles of these alkanols are similar to the values for alkanes on an OTS-coated substrate. In general, we observed that the alkanol droplets did not move under the applied thermal gradients. We suspect that evaporative effects and the higher polarity of the liquid were responsible for this behavior. It has been reported in the literature that the higher the liquid polarity, the more sensitive is the droplet motion to surface defects, a condition which typically results in significant contact angle hysteresis. Lam et al. experimented with various alkanes and alcohols on fluorinated surfaces. ${ }^{49}$ Their data clearly show that alkanols generate much larger contact angle hysteresis than alkane droplets for comparable values of the surface tension. These observations are consistent with our findings.

\section{CONCLUSION}

This work seeks to parametrize the forces governing the threshold retentive force and migration speed of small (single-component) liquid droplets propelled across a chemically homogeneous surface by thermocapillary stresses. These stresses are generated at the air-liquid interface by differentially heating the supporting substrate to produce a constant thermal gradient. The droplets always move toward the cooler side of the substrate. The migration speed was examined as a function of droplet radius, thermal gradient, and liquid parameters on two types of silanized surfaces (DTS and OTS). The grafting procedure described led to high-quality and reproducible surfaces containing a small number of defects and small contact angle hysteresis. These results were compared to predictions of a simplified hydrodynamic model for 2D thermocapillary migration including contact angle hysteresis based on a force balance at the liquid-solid interface. The agreement with the experimental data is found to be very good despite the fact that the model strictly applies to a two-dimensional droplet and that the viscosity is assumed constant. The two fitting parameters in the model are the difference in contact angle between the advancing and receding ends of the droplet and the slip coefficient used to regularize the well-known stress singularity at a moving contact line.

For a given liquid, measurements of the droplet speed, $U(R, \tau)$, as a function of the driving force, represented by the product $\tau R$, collapse onto a straight line. The horizontal intercept denotes the critical value of the retentive force required for droplet mobilization by thermocapillary forces. The linear dependence of the droplet speed on the driving force as well as the value of the threshold force for depinning are well described by the predictions of the hydrodynamic model. Further investigation of the model has revealed that contact angle hysteresis influences the speed and threshold values much more significantly than the slip length for physically meaningful parameter values. This conclusion is expected to hold true for other actuation schemes involving droplet motion whether in enclosed channels or open geometries. Studies along the lines presented here will illustrate more generally the critical nature of contact angle hysteresis in optimizing droplet speed while minimizing power requirements in microfluidic devices.

\section{ACKNOWLEDGMENTS}

The authors gratefully acknowledge financial support from the National Science Foundation (CTS and DMR), the NASA Microgravity Fluid Physics Program, and the U.S. Army TACOM-ARDEC. SMT kindly acknowledges the warm hospitality and generous support of the Moore Distinguished Scholar Program at Caltech, where final editorial changes were made.

${ }^{1}$ A. Manz, C. S. Effenhauser, N. Burggraf, D. J. Harrison, K. Seiler, and K. Fluri, J. Micromech. Microeng. 4, 257 (1994).

${ }^{2}$ S. F. Bart, L. S. Tavrow, M. Mehregany, and J. H. Lang, Sens. Actuators, A 21-23, 193 (1990).

${ }^{3}$ P. Gravesen, J. Branebjerg, and O. S. Jensen, J. Micromech. Microeng. 3, 168 (1993).

${ }^{4}$ T. S. Sammarco and M. A. Burns, AIChE J. 45, 350 (1999).

${ }^{5}$ D. C. Duffy, H. L. Gillis, J. Lin, N. F. Sheppard, and G. J. Kellogg, Anal. Chem. 71, 4669 (1999).

${ }^{6}$ J. Jang and S. S. Lee, Sens. Actuators, A 80, 84 (2000).

${ }^{7}$ W. J. J. Welters and L. G. J. Fokkink, Langmuir 14, 1535 (1998).

${ }^{8}$ J. Lee and C.-J. Kim, J. Microelectromech. Syst. 9, 171 (2000).

${ }^{9}$ M. G. Pollack, R. B. Fair, and A. D. Shenderov, Appl. Phys. Lett. 77, 1725 (2000)

${ }^{10}$ B. R. Acharya, T. Krupenkin, S. Ramachandran, Z. Wang, C. C. Huang, and J. A. Rogers, Appl. Phys. Lett. 83, 4912 (2003).

${ }^{11}$ T. Krupenkin, S. Yang, and P. Mach, Appl. Phys. Lett. 82, 316 (2003).

${ }^{12}$ R. A. Hayes and B. J. Feenstra, Nature (London) 425, 383 (2003).

${ }^{13}$ T. B. Jones, M. Gunji, M. Washizu, and M. J. Feldman, J. Appl. Phys. 89, 1441 (2001).

${ }^{14}$ A. A. Darhuber, J. M. Davis, S. M. Troian, and W. W. Reisner, Phys. Fluids 15, 1295 (2003).

${ }^{15}$ A. A. Darhuber, J. P. Valentino, J. M. Davis, S. M. Troian, and S. Wagner, Appl. Phys. Lett. 82, 657 (2003).

${ }^{16}$ A. A. Darhuber, J. P. Valentino, S. M. Troian, and S. Wagner, J. Microelectromech. Syst. 12, 873 (2003).

${ }^{17}$ P. Mach, T. Krupenkin, S. Yang, and J. A. Rogers, Appl. Phys. Lett. 81, 202 (2002).

${ }^{18}$ A. A. Darhuber, S. M. Troian, and S. Wagner, J. Appl. Phys. 91, 5686 (2002).

${ }^{19}$ A. A. Darhuber, J. Z. Chen, J. M. Davis, and S. M. Troian, Philos. Trans. R. Soc. London, Ser. A 362, 1037 (2004).

${ }^{20}$ R. H. Johnson and R. H. Dettre, J. Phys. Chem. 68, 1744 (1964).

${ }^{21}$ R. H. Dettre and R. H. Johnson, J. Phys. Chem. 69, 1507 (1965).

${ }^{22}$ R. H. Johnson and R. H. Dettre, Adv. Chem. Ser. 43, 113 (1964).

${ }^{23}$ R. H. Dettre and R. H. Johnson, Adv. Chem. Ser. 43, 137 (1964).

${ }^{24}$ Y. L. Chen, C. A. Helm, and J. N. Israelachvili, J. Phys. Chem. 95, 10736 (1991).

${ }^{25}$ Y.-T. Tseng, F.-G. Tseng, and C.-C. Chieng, 12th International Conference on Solid State Sensors, Actuators and Microsystems Digest 2003, pp. 1987-1882.

${ }^{26}$ F. Brochard, Langmuir 5, 432 (1989).

${ }^{27}$ J. B. Brzoska, F. Brochard-Wyart, and F. Rondelez, Langmuir 9, 2220 (1993).

${ }^{28}$ M. L. Ford and A. Nadim, Phys. Fluids 6, 3183 (1994).

${ }^{29}$ M. K. Smith, J. Fluid Mech. 294, 209 (1995).

${ }^{30}$ A. L. Yarin, W. Liu, and D. H. Reneker, J. Appl. Phys. 91, 2751 (2002). 
${ }^{31}$ P. G. de Gennes, Rev. Mod. Phys. 57, 827 (1985).

${ }^{32}$ N. B. Vargaftik, Handbook of Physical Properties of Liquids and Gases (Hemisphere Publishing Corp., New York, 1983). The handbook data was used to generate an expression for the variation of viscosity, $\mu[\mathrm{mPas}]$, with temperature, $T\left[{ }^{\circ} \mathrm{C}\right]$ : $\mu_{\text {decane }}(T)$ $=0.074+0.51 \exp (-T / 28.78)+0.70 \exp (-T / 104.79), \quad \mu_{\text {decane }}(T)=-0.072$ $+1.24 \exp (-T / 29.04)+1.09 \exp (-T / 143.14), \quad \mu_{\text {tetradecane }}(T)=-0.4263$ $+2.44 \exp (-T / 26.38)+1.805 \exp (-T / 186.68)$, and $\mu_{\text {hexadecane }}(T)=0.456$ $+1.91 \exp (-T / 8.971)+4.41 \exp (-T / 43.42)$.

${ }^{33}$ M. de Ruijter, P. Kölsch, M. Voué, J. De Coninck, and J. P. Rabe, Colloids Surf., A 144, 238 (1998). The experimental data for squalane in this reference was used to generate an expression for the variation in viscosity, $\mu[\mathrm{mPas}]$, with temperature, $T\left[{ }^{\circ} \mathrm{C}\right]: \mu_{\text {squalane }}(T)$ $=3.25+110.5 \exp (-T / 3.45)+75.57 \exp (-T / 23.59)$.

${ }^{34}$ J. B. Brzoska, N. Shahidzadeh, and F. Rondelez, Nature (London) 360, 719 (1992).

${ }^{35}$ J. B. Brzoska, I. B. Azouz, and F. Rondelez, Langmuir 10, 4367 (1994).

${ }^{36}$ Y. Liu, L. K. Wolf, and M. C. Messmer, Langmuir 17, 4329 (2001).

${ }^{37}$ Y. Wang and M. Lieberman, Langmuir 19, 1159 (2003).
${ }^{38}$ N. Rozlosnik, M. C. Gerstenberg, and N. B. Larsen, Langmuir 19, 1182 (2003).

${ }^{39}$ M. E. McGovern, K. M. R. Kallury, and M. Thompson, Langmuir 10, 3607 (1994).

${ }^{40}$ W. A. Zisman, Adv. Chem. Ser. 43, 1 (1964).

${ }^{41}$ C. W. Extrand and Y. Kumagai, J. Colloid Interface Sci. 191, 378 (1997).

${ }^{42}$ W. M. Deen, Analysis of Transport Phenomena (Oxford University Press, New York, 1998).

${ }^{43}$ A. W. Adamson, Physical Chemistry of Surface, 5th ed. (Wiley, New York, 1990).

${ }^{44}$ E. B. Dussan V., Annu. Rev. Fluid Mech. 11, 371 (1979).

${ }^{45}$ C. G. Ngan and E. B. Dussan V., J. Fluid Mech. 118, 27 (1982).

${ }^{46}$ W. Rose and R. W. Heins, J. Colloid Sci. 17, 39 (1962).

${ }^{47}$ H. P. Greenspan, J. Fluid Mech. 84, 125 (1978).

${ }^{48}$ C. Huh and L. E. Scriven, J. Colloid Interface Sci. 35, 85 (1971).

${ }^{49}$ C. N. C. Lam, N. Kim, D. Hui, D. Y. Kwok, M. L. Hair, and A. W. Neumann, Colloids Surf. 189, 265 (2001).

${ }^{50}$ L. H. Tanner, J. Phys. D 12, 1473 (1979). 